\title{
Randomized, double-blinded, placebo-controlled trial comparing two multimodal opioid-minimizing pain management regimens following transsphenoidal surgery
}

\author{
Deborah M. Shepherd, ACNP, Heidi Jahnke, MSN, William L. White, MD, and Andrew S. Little, MD \\ Department of Neurosurgery, Barrow Neurological Institute, St. Joseph's Hospital and Medical Center, Phoenix, Arizona
}

\begin{abstract}
OBJECTIVE Pain control is an important clinical consideration and quality-of-care metric. No studies have examined postoperative pain control following transsphenoidal surgery for pituitary lesions. The study goals were to 1) report postoperative pain scores following transsphenoidal surgery, 2) determine if multimodal opioid-minimizing pain regimens yielded satisfactory postoperative pain control, and 3) determine if intravenous (IV) ibuprofen improved postoperative pain scores and reduced opioid use compared with placebo.
\end{abstract}

METHODS This study was a single-center, randomized, double-blinded, placebo-controlled intervention trial involving adult patients with planned transsphenoidal surgery for pituitary tumors randomized into 2 groups. Group 1 patients were treated with scheduled IV ibuprofen, scheduled oral acetaminophen, and rescue opioids. Group 2 patients were treated with IV placebo, scheduled oral acetaminophen, and rescue opioids. The primary end point was patient pain scores (visual analog scale [VAS], rated $0-10$ ) for 48 hours after surgery. The secondary end point was opioid use as estimated by oral morphine equivalents (OMEs).

RESULTS Of 136 patients screened, 62 were enrolled (28 in Group 1, 34 in Group 2). The study was terminated early because the primary and secondary end points were reached. Baseline characteristics between groups were well matched except for age (Group 1, $59.3 \pm 14.4$ years; Group 2, $49.8 \pm 16.2$ years; $p=0.02$ ). Mean VAS pain scores were significantly different, with a 43\% reduction in Group $1(1.7 \pm 2.2)$ compared with Group $2(3.0 \pm 2.8 ; p<0.0001)$. Opioid use was significantly different, with a 58\% reduction in Group 1 (26.3 $\pm 28.7 \mathrm{mg}$ OME) compared with Group 2 (62.5 \pm $63.8 \mathrm{mg}$ OME; $p<0.0001)$.

CONCLUSIONS Multimodal opioid-minimizing pain-management protocols resulted in acceptable pain control following transsphenoidal surgery. IV ibuprofen resulted in significantly improved pain scores and significantly decreased opioid use compared with placebo. Postoperative multimodal pain management, including a nonsteroidal antiinflammatory medication, should be considered after surgery to improve patient comfort and to limit opioid use.

Clinical trial registration no.: NCT02351700 (clinicaltrials.gov)

- CLASSIFICATION OF EVIDENCE Type of question: therapeutic; study design: randomized, controlled trial; evidence: Class III.

https://thejns.org/doi/abs/10.3171/2016.10.JNS161355

KEY WORDS ibuprofen; pain; pituitary surgery; transsphenoidal surgery; visual analog scale

$\mathrm{P}$ OSTOPERATIVE pain is an important clinical concern and quality-of-care metric, yet it is undertreated in neurosurgical patients. ${ }^{25}$ Approximately $40 \%$ of inpatients complain of severe pain postoperatively, and only $56 \%$ of these patients indicate that their pain is well controlled. ${ }^{17}$ In addition, pain is a common cause of delayed discharge and unplanned hospital readmission. ${ }^{1,2,5,6,8,13}$,
14,19,24,26 Recently, the patient's perception of postoperative pain has been included as a domain in the Hospital Consumer Assessment of Healthcare Providers and Systems (HCAHPS) scores. Because of these numerous influences, interest in improving postoperative pain management in neurosurgery is increasing.

In neurosurgical patients, opioids are the most frequent-

ABBREVIATIONS BMI = body mass index; $C A B G=$ coronary artery bypass graft; HCAHPS = Hospital Consumer Assessment of Healthcare Providers and Systems; IV = intravenous; NSAID = nonsteroidal antiinflammatory drug; OME = oral morphine equivalent; $\mathrm{VAS}=$ visual analog scale.

SUBMITTED May 26, 2016. ACCEPTED October 10, 2016.

INCLUDE WHEN CITING Published online March 3, 2017; DOI: 10.3171/2016.10.JNS161355. 


\section{- CLASSIFICATION OF EVIDENCE}

Type of Question Therapeutic

Study Design Randomized, Controlled Trial

Evidence Class III

Although well-designed randomized controlled trials (RCTs) provide the strongest evidence in support of clinical decision making, they are extraordinarily difficult and expensive to conduct. Shepherd and colleagues have completed such a trial, and all the more remarkably they have done so within a single institution and without the extensive industry support that almost always characterizes modern RCTs. For this accomplishment, these authors deserve high praise. Their study compares pain scores (using a visual analog scale [VAS]) over the first 48 hours following transsphenoidal surgery in patients randomized to receive scheduled oral acetaminophen, rescue opioids, and either scheduled intravenous (IV) ibuprofen or placebo. They found improved pain scores and reduced opioid use in the ibuprofen group. The study raises a number of interesting statistical issues. The authors' study contains most of the elements of a Class I trial: randomization, allocation concealment, masked outcome assessment, pre-specified primary outcome measure, minimal loss to follow-up, and intent-to-treat analysis. Unfortunately, some characteristics of the 2 study arms (Table 1) differ appreciably. For example, mean age differs by 9.5 years. Ethnicity, procedure type, tumor type, and initial postoperative VAS scores are also substantially different between groups. One might object that only age is "significantly different" as judged by $p$ values, but this objection misrepresents the meaning of $p$ values, which only express the likelihood that the difference present between the 2 treatment groups is due to chance. If the randomization procedure in this trial was not corrupted, we know that any difference between groups must have occurred by chance. The $p$ values are not so important here,

ly used class of medications to control pain..$^{9,17,23}$ However, opioids are associated with adverse effects such as constipation, altered mental status, and respiratory depression. Recently, multimodal strategies have been shown to improve pain control and limit opioid-induced adverse effects. $3,7,12,20,27$ The rationale for a multimodal approach is that a combination of opioids and nonopioid analgesics, which work by different mechanisms, will potentiate pain relief and limit adverse reactions. Intravenous (IV) nonsteroidal antiinflammatory drugs (NSAIDs) such as ibuprofen have been used as part of multimodal paincontrol strategies in orthopedic surgery and abdominal surgery. ${ }^{15,21}$ IV ibuprofen (Caldolor, Cumberland Pharmaceuticals, Inc.) was approved in 2009 for management of mild to moderate pain and, in combination with opioids, for severe pain.

Postoperative pain following transsphenoidal surgery for pituitary lesions has not been studied. Because transsphenoidal surgery uses a minimal-access approach with limited tissue disruption, patients undergoing this procedure are good candidates for an evaluation of the impact of multimodal opioid-minimizing strategies. The aims of this study were 1) to report postoperative pain scores fol- and many journals will not allow $p$ values in tables of patient characteristics from RCTs. The important issue is whether the difference between groups is clinically meaningful. The authors do attempt to control for age in their analysis, but do not control for potentially more important differences such as initial VAS score. The $p$ values may also be given more weight than they deserve in the interpretation of outcomes. While the difference in mean pain scores (the study's primary outcome measure) is unlikely to be due to chance $(p<0.0001)$, the magnitude of the difference in scores is small: 1.3 ( $95 \%$ confidence interval 0.02-2.6). Again, clinical judgment is required to determine whether this difference is important. Moreover, the difference does not reach the "2-point mean difference" that the authors established a priori as their definition of a successful intervention. So, in fact, the study may not be as positive as the authors claim, because it failed to meet its pre-specified end point. Again, the authors should be praised for their attempts to conduct this RCT.

$$
\begin{array}{r}
\text { - Michael Glantz, MD } \\
\text { Hershey Medical Center } \\
\text { Hershey, Pennsylvania }
\end{array}
$$

\section{Authors' Response \\ We welcome the level of evidence comments as a way to strive to improve the quality of clinical trials in neurosurgery. The reviewer has provided a valuable perspective. Our only disagreement with these comments is that it is not advisable to control for initial VAS pain score because patients have already started receiving the experimental intervention during surgery, and the initial VAS score has therefore already been influenced by this intervention. The initial VAS score in the ibuprofen group is expectedly lower than the VAS score in the placebo group because the patients in the ibuprofen group have received ibuprofen during surgery.}

lowing transsphenoidal surgery, 2) to determine if multimodal opioid-minimizing pain regimens yielded satisfactory postoperative pain control, and 3) to determine if IV ibuprofen improved postoperative pain scores and reduced opioid use compared with placebo.

\section{Methods}

\section{Trial Design}

The study was a single-center, randomized, doubleblinded intervention trial comparing outcomes in 2 groups of patients treated with multimodal pain management protocols following transsphenoidal surgery for pituitary lesions. The trial was conducted between February 1, 2015, and March 1, 2016. The study was approved by the Institutional Review Board of St. Joseph's Hospital and Medical Center, Phoenix, Arizona, and was registered with clinicaltrials.gov (no. NCT02351700).

\section{Study Population and Inclusion and Exclusion Criteria}

Study participants included adult patients undergoing endonasal transsphenoidal surgery for pituitary adenoma. 


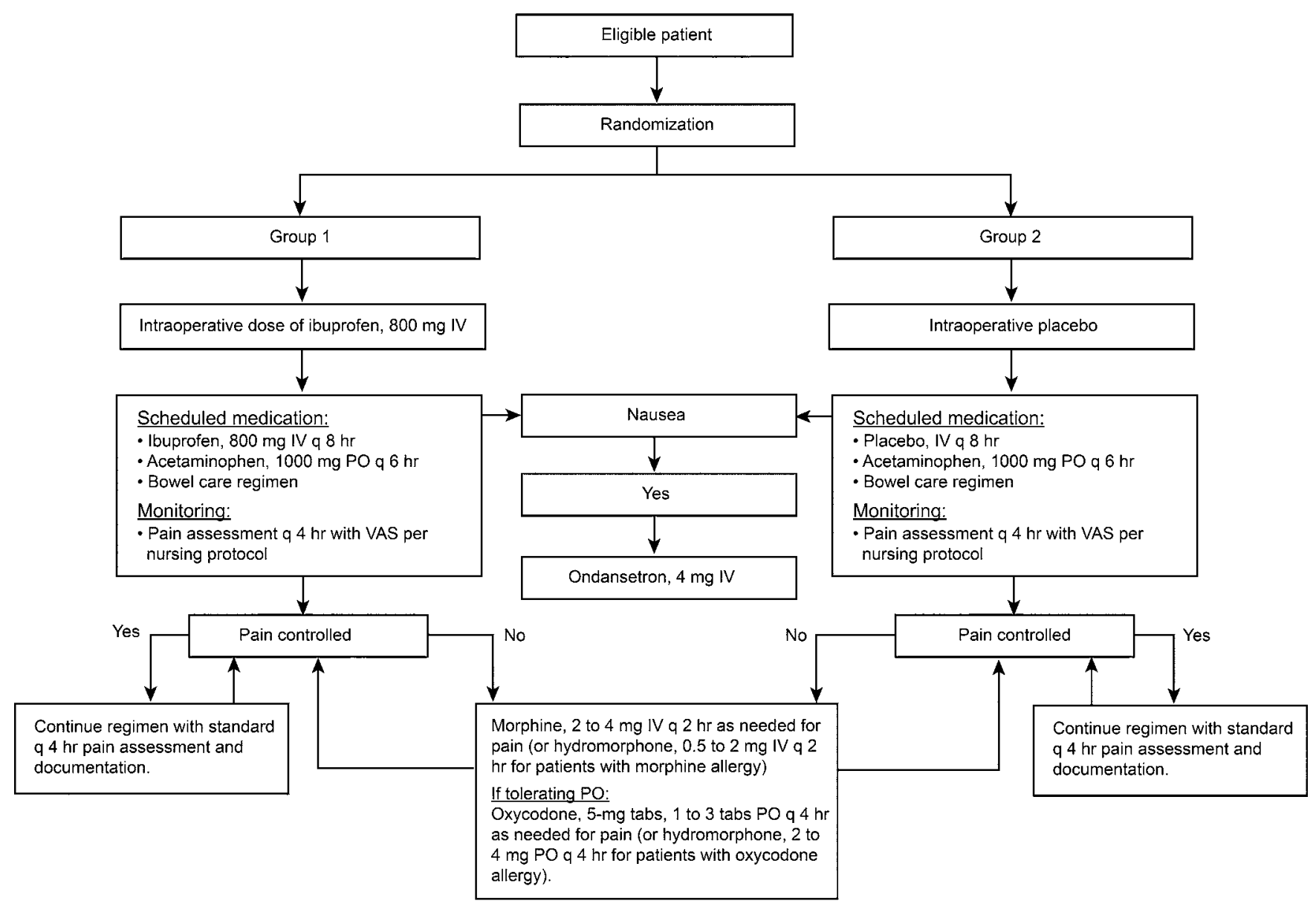

FIG. 1. Flow diagram of study interventions. $P O=$ by mouth; $q=$ every.

Potential patients were identified in the Barrow Pituitary Clinic at Barrow Neurological Institute and screened for eligibility by the research nurse (H.J.). Eligible patients included adult patients who were aged 18 to 80 years, who were English speaking, and who had a body mass index (BMI) greater than 19 and less than $40 \mathrm{~kg} / \mathrm{m}^{2}$. The study excluded patients with renal insufficiency (creatinine $>2.0$ $\mathrm{mg} / \mathrm{dl}$ ); allergy or intolerance to the study drugs or opioids; a history of opioid tolerance or abuse; peptic ulcer disease or intestinal bleeding; cardiac disease or liver disease (liver function tests greater than 3 times the upper limit of normal); and patients who were pregnant, incarcerated, or unwilling to participate in the study. Informed consent was obtained by the principal investigator or designee.

\section{Interventions}

Study interventions are summarized in Fig. 1. Group 1 patients were treated with scheduled IV ibuprofen, scheduled oral acetaminophen, and rescue opioids. The first dose of ibuprofen was administered intraoperatively at the time the sella turcica was opened. Group 2 patients were treated with IV saline placebo, scheduled oral acetaminophen, and rescue opioids. Saline placebo was administered according to the same schedule as IV ibuprofen in Group 1.

\section{Outcome Measures}

The primary end point of the study was patient pain scores measured on a scale of 0-10 (visual analog scale [VAS]) every 4 hours for the first 48 hours after surgery. The secondary end point was the estimated oral morphine equivalent (OME), used for breakthrough pain in the first 48 hours after surgery. Several supplementary outcomes were also tracked, including length of stay following surgery, use of antiemetics, and number of bowel movements during hospitalization.

\section{Sample Size}

Fifty treated patients in each group were required to detect a 2-point mean difference on the 11-point (0-10) VAS, with a standard deviation of 3.2 for the placebo group and 3.5 for the treatment group, with $\alpha$ set at 0.05 and $90 \%$ power.

\section{Stopping Guidelines}

A planned interim analysis after approximately 60 patients were enrolled was established in the study protocol. Stopping guidelines for the study included the study reaching significance with its primary end point or upon detection of an unacceptable number of adverse events. A 


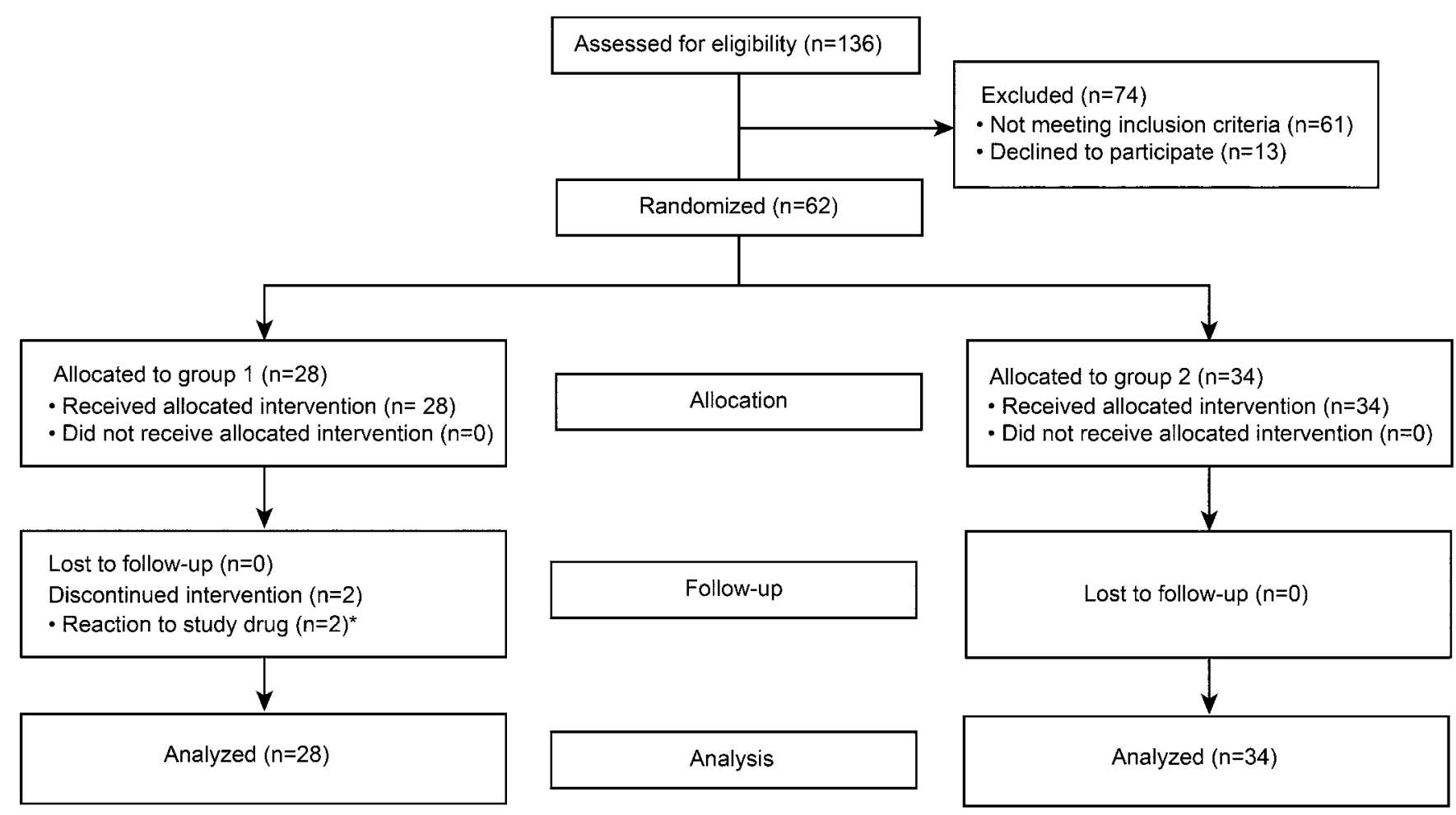

*Two patients each received 3 doses of the study medication; 1 discontinued study drug due to burning at the IV site and 1 discontinued because of persistent hyperkalemia. Both patients were included in the intent-to-treat analysis.

FIG. 2. Consolidated Standards of Reporting Trials (CONSORT) flow diagram.

Data Review Committee consisting of a research pharmacist, an independent research nurse, a neurologist with extensive experience in clinical trial development, and the principal investigator (A.S.L.) reviewed the study data.

\section{Randomization}

Patients were randomized in a 1:1 ratio with blinded treatment assignment. The patients were randomized using a computer-generated list of random numbers from www.random.org. The randomized list was placed with an ordered list of numbers from 1 to 100 . Odd numbers were assigned to Group 1 and even numbers to Group 2. For example, if the first number drawn was 61, that patient was assigned to Group 1. The research nurse generated the random number sequence and performed the blinded assignment. Patients, family members, bedside nurses, and providers were blinded to treatment assignment. The treatment assignment was known by the research nurse and a research pharmacist.

\section{Statistical Analysis}

The cohort was described using frequencies with percentages for categorical variables and means with standard deviations for continuous variables. Patient data were analyzed using an intent-to-treat analysis. Chi-squared tests and independent-samples t-tests were used as appropriate to assess differences between groups. A linear mixed model with time point as the repeated measure, treatment group as the factor, age as a covariate, and VAS score as the dependent variable was used to assess the overall mean difference in patients' self-reported VAS scores. Independent-samples t-tests were also used to assess mean differences in patients' self-reported pain at each time point. SPSS Statistics for Windows (version 22.0, IBM Corp.) was used for analyses, and $\mathrm{p}$ values < 0.05 were considered statistically significant.

\section{Results}

Of 136 patients screened, 74 were excluded from enrollment in the study (Fig. 2). Of the 74 patients excluded, 61 did not meet eligibility criteria and 13 declined to participate. The most common reasons for excluding patients from the study were chronic opioid use $(\mathrm{n}=14)$, age $(\mathrm{n}=$ $10)$, BMI $>40 \mathrm{~kg} / \mathrm{m}^{2}(\mathrm{n}=10)$, non-English speaking ( $\mathrm{n}$ $=4)$, history of cardiovascular disease $(\mathrm{n}=4)$, history of gastrointestinal bleeding $(n=4)$, history of intolerance or allergy to study drug $(n=3)$, history of hepatitis $(n=3)$, renal failure $(n=2)$, and other $(n=7)$. In total, 62 patients were randomized, with 28 in Group 1 and 34 in Group 2 . The study was terminated early because the planned interim analysis demonstrated that the primary end point had been reached. A comparison of patient demographics (Table 1) indicated that patients in Group 1 (59.3 \pm 14.4 years) were significantly older than patients in Group 2 $(49.8 \pm 16.2$ years; $p=0.02)$; otherwise, the 2 groups were not significantly different in demographics.

\section{Primary and Secondary Outcomes}

Both pain protocols resulted in acceptable postopera- 
TABLE 1. Patient demographics

\begin{tabular}{|c|c|c|c|}
\hline Variable & Group 1 & Group 2 & $p$ Value \\
\hline No. of patients & 28 & 34 & \\
\hline Age (yrs) & $59.3 \pm 14.4$ & $49.8 \pm 16.2$ & 0.02 \\
\hline Sex & & & $>0.99$ \\
\hline Male & $15(53.6)$ & $18(52.9)$ & \\
\hline Female & $13(46.4)$ & $16(47.1)$ & \\
\hline Ethnicity & & & 0.19 \\
\hline African American & 0 & $1(2.9)$ & \\
\hline Asian/Pacific Islander & 0 & $2(5.9)$ & \\
\hline Hispanic & $1(3.6)$ & $6(17.6)$ & \\
\hline Middle Eastern & $1(3.6)$ & 0 & \\
\hline Native American & 0 & $1(2.9)$ & \\
\hline White & $26(92.9)$ & $24(70.6)$ & \\
\hline $\mathrm{BMI}\left(\mathrm{kg} / \mathrm{m}^{2}\right)$ & $30.1 \pm 4.9$ & $28.9 \pm 5.7$ & 0.42 \\
\hline Tumor size (mm) & $24.2 \pm 11.5$ & $20.9 \pm 12.1$ & 0.29 \\
\hline Prior transsphenoidal surgery & $1(3.6)$ & $3(8.8)$ & 0.62 \\
\hline Procedure type & & & 0.32 \\
\hline Endoscopic & $11(39.3)$ & $18(52.9)$ & \\
\hline Microscopic & $17(60.7)$ & $16(47.1)$ & \\
\hline Tumor type & & & 0.12 \\
\hline Nonfunctioning adenoma & $22(78.6)$ & $20(58.8)$ & \\
\hline Functioning adenoma & $6(21.4)$ & $14(41.2)$ & \\
\hline
\end{tabular}

Values are presented as number (\%) of patients unless indicated otherwise. Mean values are given \pm SD.

tive pain, but pain scores were significantly lower $(43 \%$ reduction) in Group 1 (ibuprofen, acetaminophen, opioids; mean VAS score $1.7 \pm 2.2$ ) compared with Group 2 (placebo, acetaminophen, opioids; mean VAS score $3.0 \pm 2.8$, $\mathrm{p}<0.0001$; Fig. 3). Pain scores in both groups tended to improve over time (Fig. 4). Age was included as a covariate in the analysis because of the significant difference in

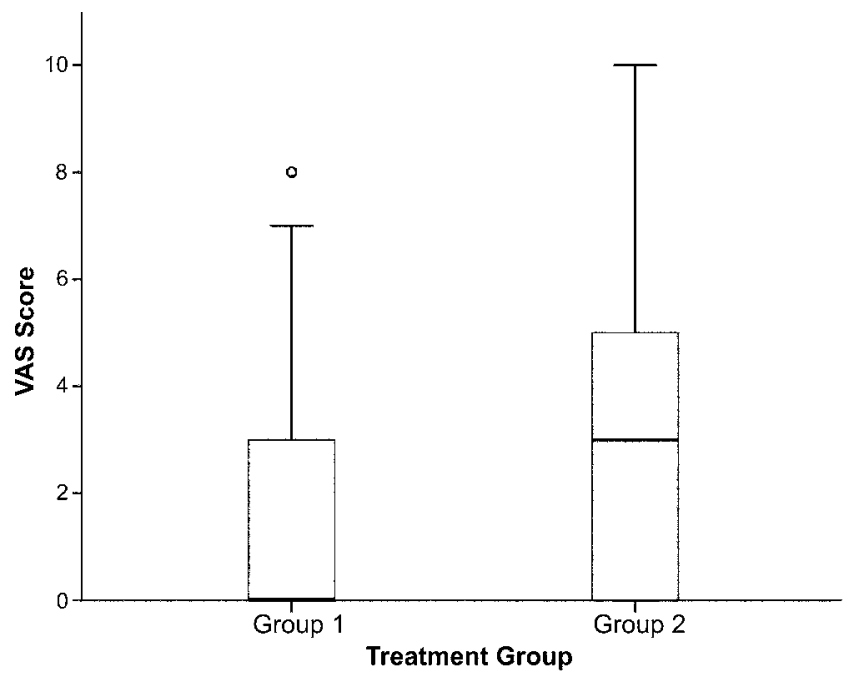

FIG. 3. Box plots of mean patient VAS pain scores following transsphenoidal surgery $(p<0.0001)$. Outliers are presented as open circles. Horizontal line in bars represents the median.

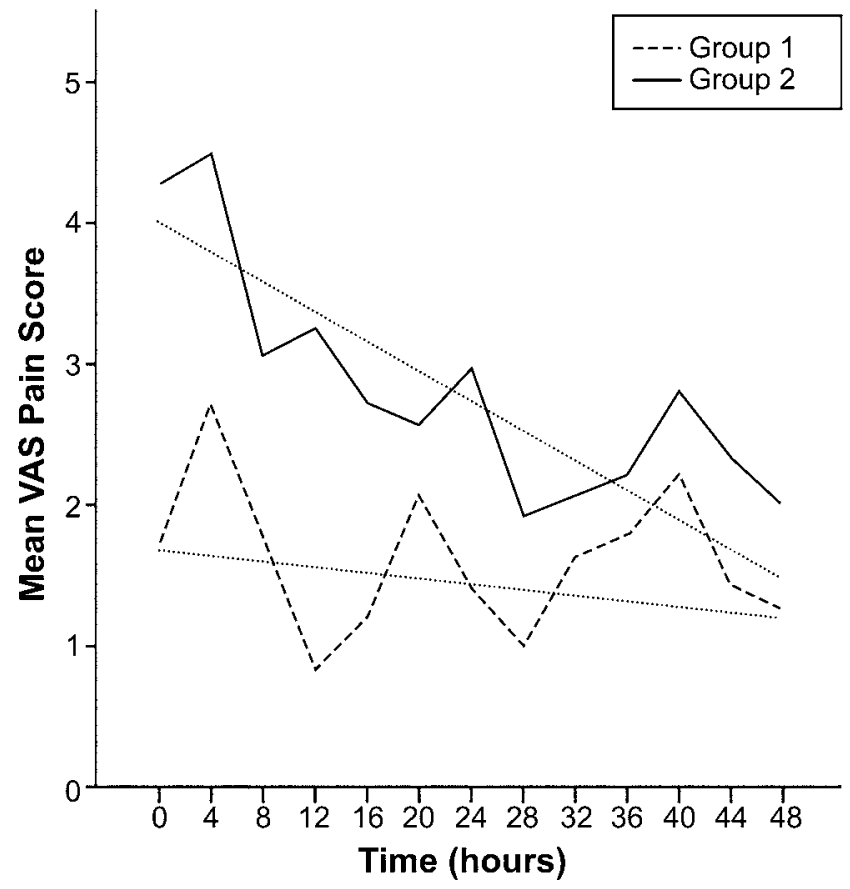

FIG. 4. Line graph with regression lines (dotted lines) of mean VAS pain scores over time after transsphenoidal surgery.

mean age between groups. Opioid use was also significantly lower (58\% reduction) in Group $1(26.3 \pm 28.7 \mathrm{mg}$ OME) than in Group 2 (62.5 $\pm 63.8 \mathrm{mg}$ OME, $\mathrm{p}<0.0001$; Fig. 5). Study outcomes are summarized in Table 2.

\section{Supplementary Outcomes}

For the supplementary end points that were evaluated (Table 2), there were no differences between the 2 groups in the use of antiemetics $(\mathrm{p}=0.92)$, number of bowel movements $(p>0.99)$, or length of stay $(p=0.07)$. The

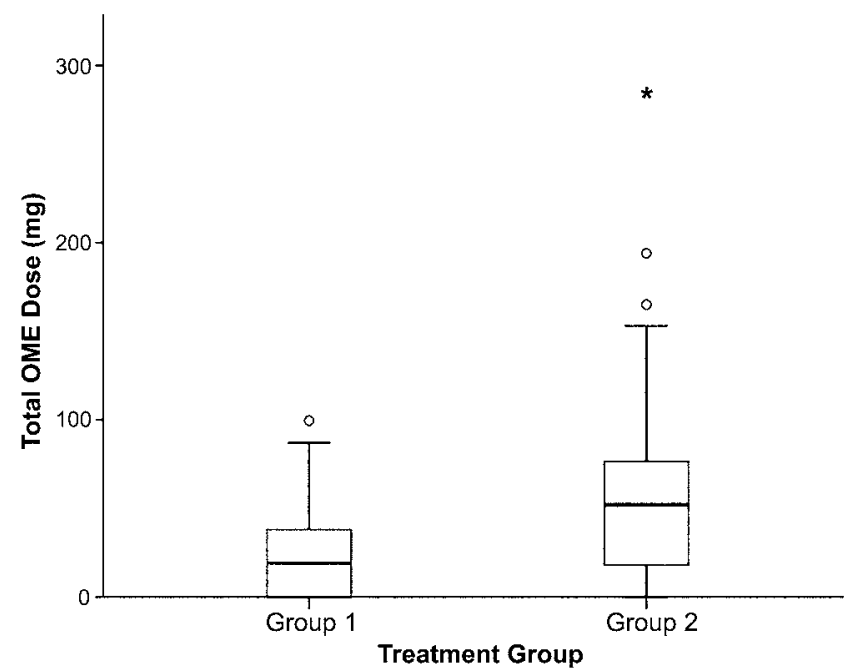

FIG. 5. Box plots of mean OME use following transsphenoidal surgery ( $p$ $<0.0001$ ). Outliers are presented as open circles, and extreme outliers as an asterisk. Horizontal line in bars represents the median. 
TABLE 2. Summary of study outcomes

\begin{tabular}{cccc}
\hline End Point & $\begin{array}{c}\text { Group 1 } \\
\text { (ibuprofen) }\end{array}$ & $\begin{array}{c}\text { Group 2 } \\
\text { (placebo) }\end{array}$ & p Value \\
\hline Primary & & & \\
\hline Pain score & $1.7 \pm 2.2$ & $3.0 \pm 2.8$ & $<0.0001$ \\
\hline Secondary & & & \\
\hline OME use (mg) & $26.3 \pm 28.7$ & $62.5 \pm 63.8$ & $<0.0001$ \\
\hline Supplementary & & & \\
\hline Antiemetic use $(\mathrm{mg})^{*}$ & $9.8 \pm 9.1$ & $10.1 \pm 6.6$ & 0.92 \\
\hline $\begin{array}{c}\text { Patients w/ } \geq 1 \text { bowel } \\
\text { movement, no. }(\%)\end{array}$ & $9(32)$ & $12(35)$ & $>0.99$ \\
\hline Length of stay (hrs) & & & \\
\hline All patients & $71.3 \pm 47.5$ & $51.7 \pm 30.3$ & 0.07 \\
\hline 1 surgery only & $53.5 \pm 16.5$ & $48.1 \pm 21.5$ & 0.31 \\
\hline
\end{tabular}

Values are presented as means \pm SD unless indicated otherwise.

* Antiemetic used was intravenous ondansetron.

primary drivers of length of stay were whether the patient underwent elective reoperation for residual pituitary tumor during the same hospital admission and intraoperative complications. In Group 1, only 3 patients underwent an elective second-stage surgery for residual tumor and 1 patient suffered a posterior cerebral artery infarct from iatrogenic injury during surgery. In Group 2, only 1 patient underwent reoperation. When considering only those patients who had undergone 1 surgery, the difference in mean length of stay was 5 hours, which was not statistically significant $(\mathrm{p}=0.31)$.

\section{Adverse Events Related to Study Drug}

There were 2 adverse events likely related to the study drug. One patient complained of burning at the IV site during the third dose of ibuprofen, and 1 patient developed postoperative hyperkalemia. No other cause of hyperkalemia was identified. Both patients were included in the intent-to-treat analysis described above.

\section{Bleeding Complications}

There were no bleeding complications likely to be related to the study drug as determined by the Data Review Committee. One patient who received ibuprofen (Group 1) developed rectal bleeding from previously undiagnosed diverticulosis 7 days after completion of the study. The Data Review Committee concluded that the study drug was unlikely to be related to the bleeding event because the patient had an underlying pathology that can cause rectal bleeding, and because study drug administration was complete 7 days prior to the event. In the placebo group (Group 2), there were 2 cases of delayed epistaxis treated with nasal packing. There were no cases of epistaxis in Group 1.

\section{Discussion}

In this article, we present the results of the first study to examine pain control following transsphenoidal surgery. We examined the impact of 2 multimodal pain-manage- ment regimens on pain scores and opioid use. The study yielded several notable findings. Our first goal was to document patient pain following transsphenoidal surgery, as this has not been previously published. We learned that pain scores following surgery were modest, and most patients reported good pain control with both opioid-minimizing protocols. The mean VAS pain score was "mild" $(1.7 \pm 2.2)$ in Group 1 (acetaminophen, ibuprofen, and rescue opioids) and "nagging" (3.0 \pm 2.8$)$ in Group 2 (acetaminophen, rescue opioids) during the first 48 hours following surgery. Pain control tended to improve over time in both groups. The second aim of the study was to determine if IV NSAIDs (i.e., ibuprofen) improved pain control and limited opioid use compared with placebo. IV ibuprofen significantly reduced patient pain (43\% reduction in VAS pain score) and rescue opioid use (58\% reduction in OME use). The study was stopped early, according to protocol, because of the dramatic and statistically significant differences in the primary and secondary end points between groups. We also examined several other outcome measures, such as constipation and use of antiemetics, but did not find a significant difference between groups. This study supports the use of multimodal pain control following transsphenoidal surgery.

\section{Study Generalizability}

The results of the study are generalizable to the typical population of patients undergoing transsphenoidal surgery for pituitary lesions. The study included adult patients of various ages with both functioning and nonfunctioning pituitary tumors. However, several types of patients who were excluded from the study should be kept in mind. First, because IV ibuprofen is contraindicated in patients with a history of coronary artery bypass graft (CABG) and may increase the risk of thromboembolic events, patients with a history of CABG or those believed to be at increased risk for stroke were excluded from the study. Furthermore, because acetaminophen is hepatotoxic, we excluded patients with abnormal liver function tests or a history of cirrhosis. We also excluded patients who were tolerant to opioids. Therefore, our study does not address whether opioid-limiting pain regimens are helpful in this subset of challenging patients. Finally, we studied ibuprofen as an adjunct to opioids; other NSAIDs that might improve multimodal pain control, such as ketorolac (which is less expensive than IV ibuprofen), were not evaluated. Therefore, our results do not necessarily generalize to other oral or IV NSAIDs.

\section{Study Limitations}

One limitation of the study is that the groups were not balanced with respect to patient age. Therefore, we analyzed the pain scores, including age as a covariate, to account for this difference. However, it is unclear how differences in age between groups might have impacted the results. The other limitation of this study is that it was not powered to detect rare bleeding complications or sellar hematoma. Ibuprofen is a COX-1 inhibitor that may cause platelet dysfunction. Interestingly, NSAIDs have been safely used in other neurosurgical populations, including patients undergoing spine surgery and craniotomy. ${ }^{10,11,25}$ 
For example, in a meta-analysis of 17 spine surgery trials, there was no increase in adverse events among patients receiving NSAIDs compared with the group receiving opioids alone. ${ }^{10}$ In a study that included ketorolac as part of a multimodal strategy following craniotomy and spine procedures, there were no cases of intracerebral hemorrhage. ${ }^{25}$ In our study, there were no cases of intracerebral hemorrhage and no sinonasal bleeding complications in the ibuprofen group, whereas there were 2 cases of epistaxis in the control group. Moeller et al. ${ }^{16}$ studied the use of ketorolac following endoscopic sinus surgery and did not note any cases of postoperative epistaxis. An integrated analysis of safety involving 1220 patients identified more adverse events in control patients than in patients receiving ibuprofen. ${ }^{22}$ Whether ibuprofen and placebo were administered preoperatively or intraoperatively, surgical site hemorrhage did not differ between the 2 groups $(5.6 \%$ for ibuprofen and $6.7 \%$ for placebo). ${ }^{15,22}$ Gastrointestinal bleeding or perforations occur in $1 \%$ of patients treated for 3-6 months and in 2\%-4\% treated for 1 year $(\mathrm{Cal}-$ dolor package insert, Cumberland Pharmaceuticals, Inc.). Because the incidence of bleeding complications and other adverse events is known to be related to dose and duration of use, it is prudent to prescribe the lowest effective dose for the shortest period of time necessary and to avoid using more than 1 NSAID at a time.

\section{Future Directions}

This study firmly establishes the role of multimodal analgesia following transsphenoidal surgery by studying the use of scheduled acetaminophen and ibuprofen and asneeded opioids. Recently, researchers have studied other opioid adjuncts, such as the gabapentinoids (i.e., pregabalin and gabapentin), in neurosurgical procedures and have noted improvement in postoperative pain control and sleep. ${ }^{4,18,20}$ Studying the impact of the gabapentinoids in transsphenoidal surgery patients is a useful future direction.

\section{Conclusions}

We present the results of a randomized, placebo-controlled, double-blinded study that evaluated the efficacy of 2 multimodal opioid-minimizing pain treatment strategies following transsphenoidal surgery for pituitary tumors. The primary findings of the study are that although both treatment arms resulted in acceptable postoperative pain scores, the outcomes in the group utilizing IV ibuprofen and acetaminophen were superior to those in the placebo and acetaminophen group, demonstrating a $43 \%$ reduction in pain scores. The same group also used 58\% fewer opioids than the placebo group. This study provides evidence that multimodal pain-control strategies improve postoperative pain control and reduce opioid use in patients undergoing transsphenoidal surgery.

\section{Acknowledgments}

We thank Dr. Kristina Chapple for biostatistical assistance and the Neuroscience Publications staff of Barrow Neurological Institute for assistance with manuscript preparation. An educational grant to fund the research nurse time was provided by the Barrow Neurological Foundation.

\section{References}

1. Breivik H: Postoperative pain management: why is it difficult to show that it improves outcome? Eur J Anaesthesiol 15:748-751, 1998

2. Breivik H, Stubhaug A: Management of acute postoperative pain: still a long way to go! Pain 137:233-234, 2008

3. Brown AK, Christo PJ, Wu CL: Strategies for postoperative pain management. Best Pract Res Clin Anaesthesiol 18:703-717, 2004

4. Burke SM, Shorten GD: Perioperative pregabalin improves pain and functional outcomes 3 months after lumbar discectomy. Anesth Analg 110:1180-1185, 2010

5. Capdevila X, Barthelet Y, Biboulet P, Ryckwaert Y, Rubenovitch J, d'Athis F: Effects of perioperative analgesic technique on the surgical outcome and duration of rehabilitation after major knee surgery. Anesthesiology 91:8-15, 1999

6. Carr DB, Goudas LC: Acute pain. Lancet 353:2051-2058, 1999

7. Ekstein MP, Weinbroum AA: Immediate postoperative pain in orthopedic patients is more intense and requires more analgesia than in post-laparotomy patients. Pain Med 12:308313,2011

8. Gust R, Pecher S, Gust A, Hoffmann V, Böhrer H, Martin E: Effect of patient-controlled analgesia on pulmonary complications after coronary artery bypass grafting. Crit Care Med 27:2218-2223, 1999

9. Jeffrey HM, Charlton P, Mellor DJ, Moss E, Vucevic M: Analgesia after intracranial surgery: a double-blind, prospective comparison of codeine and tramadol. Br J Anaesth 83:245249, 1999

10. Jirarattanaphochai K, Jung S: Nonsteroidal antiinflammatory drugs for postoperative pain management after lumbar spine surgery: a meta-analysis of randomized controlled trials. J Neurosurg Spine 9:22-31, 2008

11. Jirarattanaphochai K, Thienthong S, Sriraj W, Jung S, Pulnitiporn A, Lertsinudom S, et al: Effect of parecoxib on postoperative pain after lumbar spine surgery: a bicenter, randomized, double-blinded, placebo-controlled trial. Spine (Phila Pa 1976) 33:132-139, 2008

12. Joshi GP: Multimodal analgesia techniques for ambulatory surgery. Int Anesthesiol Clin 43:197-204, 2005

13. Joshi GP, Ogunnaike BO: Consequences of inadequate postoperative pain relief and chronic persistent postoperative pain. Anesthesiol Clin North America 23:21-36, 2005

14. Katz J, Jackson M, Kavanagh BP, Sandler AN: Acute pain after thoracic surgery predicts long-term post-thoracotomy pain. Clin J Pain 12:50-55, 1996

15. Kroll PB, Meadows L, Rock A, Pavliv L: A multicenter, randomized, double-blind, placebo-controlled trial of intravenous ibuprofen (i.v.-ibuprofen) in the management of postoperative pain following abdominal hysterectomy. Pain Pract 11:23-32, 2011

16. Moeller C, Pawlowski J, Pappas AL, Fargo K, Welch K: The safety and efficacy of intravenous ketorolac in patients undergoing primary endoscopic sinus surgery: a randomized, double-blinded clinical trial. Int Forum Allergy Rhinol 2:342-347, 2012

17. Quiney N, Cooper R, Stoneham M, Walters F: Pain after craniotomy. A time for reappraisal? Br J Neurosurg 10:295299, 1996

18. Ryzenman JM, Pensak ML, Tew JM Jr: Headache: a quality of life analysis in a cohort of 1,657 patients undergoing acoustic neuroma surgery, results from the acoustic neuroma association. Laryngoscope 115:703-711, 2005

19. Shea RA, Brooks JA, Dayhoff NE, Keck J: Pain intensity and postoperative pulmonary complications among the elderly after abdominal surgery. Heart Lung 31:440-449, 2002

20. Shimony N, Amit U, Minz B, Grossman R, Dany MA, Gonen L, et al: Perioperative pregabalin for reducing pain, 
analgesic consumption, and anxiety and enhancing sleep quality in elective neurosurgical patients: a prospective, randomized, double-blind, and controlled clinical study. J Neurosurg [epub ahead of print February 12, 2016. DOI: 10.3171/2015.10.JNS151516]

21. Singla N, Rock A, Pavliv L: A multi-center, randomized, double-blind placebo-controlled trial of intravenous-ibuprofen (IV-ibuprofen) for treatment of pain in post-operative orthopedic adult patients. Pain Med 11:1284-1293, 2010

22. Southworth SR, Woodward EJ, Peng A, Rock AD: An integrated safety analysis of intravenous ibuprofen $\left(\right.$ Caldolor $\left.^{\circledR}\right)$ in adults. J Pain Res 8:753-765, 2015

23. Stoneham MD, Cooper R, Quiney NF, Walters FJ: Pain following craniotomy: a preliminary study comparing PCA morphine with intramuscular codeine phosphate. Anaesthesia 51:1176-1178, 1996

24. Tasmuth T, Kataja M, Blomqvist C, von Smitten K, Kalso E: Treatment-related factors predisposing to chronic pain in patients with breast cancer-a multivariate approach. Acta Oncol 36:625-630, 1997

25. Titsworth WL, Abram J, Guin P, Herman MA, West J, Davis NW, et al: A prospective time-series quality improvement trial of a standardized analgesia protocol to reduce postoperative pain among neurosurgery patients. J Neurosurg [epub ahead of print March 11, 2016. DOI: 10.3171/2015.10. JNS15698]

26. Tsui SL, Law S, Fok M, Lo JR, Ho E, Yang J, et al: Postoper- ative analgesia reduces mortality and morbidity after esophagectomy. Am J Surg 173:472-478, 1997

27. Wu CL, Raja SN: Treatment of acute postoperative pain. Lancet 377:2215-2225, 2011

\section{Disclosures}

Dr. Little is an investor in Kogent Surgical, LLC. The study drug (IV ibuprofen [Caldolor]) was provided by Cumberland Pharmaceuticals, Inc. Cumberland Pharmaceuticals had no role in study design, data collection, data analysis, or manuscript preparation. None of the authors have any financial relationship with the company.

\section{Author Contributions}

Conception and design: Little, Shepherd. Acquisition of data: Jahnke. Analysis and interpretation of data: Little, Shepherd. Drafting the article: Little. Critically revising the article: Shepherd, White. Reviewed submitted version of manuscript: all authors. Administrative/technical/material support: Jahnke. Study supervision: Little.

\section{Correspondence}

Andrew S. Little, c/o Neuroscience Publications, Barrow Neurological Institute, St. Joseph's Hospital and Medical Center, $350 \mathrm{~W}$ Thomas Rd., Phoenix, AZ 85013. email: neuropub@dignityhealth. org. 